\title{
Heuristic Algorithms for Power Amplifier Behavioral Modeling
}

\author{
P. L. Gilabert, D. D. Silveira, G. Montoro, M. E. Gadringer, and E. Bertran, Senior Member, IEEE
}

\begin{abstract}
This letter presents the use of two heuristic search algorithms, named simulated annealing and genetic algorithms, for the extraction of power amplifier (PA) behavioral model parameters. Their application in this letter consists in determining the memory length and the most significant delays of the considered model structure. Two PA behavioral models have been considered: an augmented nonlinear moving average model and a nonlinear auto-regressive moving average model. By using WCDMA signals measured from a three-stage LDMOS class AB PA, both PA models were extracted. Finally, results presenting the advantages of using these heuristic search algorithms are provided.
\end{abstract}

Index Terms-Behavioral models, genetic algorithm, heuristic search, power amplifier (PA), simulated annealing.

\section{INTRODUCTION}

B LACK-BOX or behavioral models describe the power amplifier (PA) input-output sampled signals relation which characterizes its nonlinear dynamics. These models are used in digital predistortion (DPD) linearization applications to identify the inverse of the PA nonlinear function and also its frequency dependence or memory effects [1]. When designing DPD at least three major issues have to be taken into account: the need for accurate PA behavioral modeling capable to achieve the demanded requirements (in terms of BW, PAPR, and memory effects reproduction), an efficient model inversion procedure and an efficient implementation in the digital processing devices [digital signal processors (DSPs) or field-programmable gate arrays (FPGAs)] without an excessive computational cost.

Often used behavioral models reported in literature are the neural networks and simplified versions of the general Volterra series. Several of these behavioral models were theoretically and practically described and compared in [2] and [3] respectively. Neural networks algorithms perform cross combinations of the input data within the hidden layers. As no optimization

Manuscript received February 12, 2007; revised June 5, 2007. This work was supported in part by the Spanish Government (MEC) under Project TEC2005-07985-C03-02 and by the EU Network TARGET “Top Amplifier Research Group in a European Team" IST-1-507893-NOE.

P. L. Gilabert, G. Montoro, and E. Bertran are with the Department of Signal Theory and Communications, Technical University of Catalonia (UPC), Barcelona 08860, Spain (e-mail: plgilabert@tsc.upc.edu; montoro@tsc.upc.edu; bertran@tsc.upc.edu).

D. D. Silveira and M. E. Gadringer are with the Institute of Electrical Measurements and Circuit Design, Vienna University of Technology (TUW), Vienna A-1040, Austria (e-mail: daniel.silveira@tuwien.ac.at; michael.gadringer@tuwien.ac.at).

Color versions of one or more of the figures in this letter are available at http://ieeexplore.ieee.org.

Digital Object Identifier 10.1109/LMWC.2007.905628 on the importance of these input data cross combinations in hidden layers is performed, neural networks are often inefficient. Volterra models have the disadvantage of using a huge number of parameters when considering higher order kernel extractions which can lead to an inaccurate identification. A common strategy is to use reduced versions of the general Volterra series consisting in structures of linear time-invariant filters and memoryless nonlinearities (Hammerstein, Wiener and its three-box modeling variants). In order to minimize these behavioral models complexity (searching computationally efficient behavioral models) some studies have been aimed at determining the minimum memory length needed to accurately characterize the PA behavioral model, as in [4].

This letter presents the use of two heuristic search algorithms, the simulated annealing and the genetic algorithms, to deal with the trade-off between behavioral models complexity (memory length and significant sparse delays) and accuracy. Two behavioral models have been considered to prove the usefulness of these algorithms: an augmented nonlinear moving average (NMA) model and a nonlinear auto-regressive moving average (NARMA) model.

\section{Power Amplifier Behavioral Models}

In this section, the considered behavioral models will be briefly explained. From now on, $x(k)$ and $y(k)$ represent the complex envelope (low-pass equivalent) input and output signals of the considered PA; and $\tau$ 's are the most suitable delays for describing the PA model.

\section{A. Augmented Nonlinear Moving Average Models}

The augmented NMA PA behavioral model is an extension of the NMA model (or also called memory polynomial model, [3]) which introduces pairs of delayed samples of the input $(x(k-$ $\left.\left.\tau_{i}\right), x\left(k-\tau_{j}\right)\right)$ up to $p$ th-order, to improve nonlinear memory modeling.

The augmented NMA model structure is depicted in Fig. 1 and its input-output relation can be expressed as

$$
\hat{y}_{\operatorname{AugNMA}}(k)=\sum_{i=0}^{N} \sum_{j=i}^{N} f_{i j}\left(x\left(k-\tau_{i}\right), x\left(k-\tau_{j}\right)\right)
$$

where $f_{i j}(\cdot)$ are static nonlinear functions implemented using polynomials

$$
f_{i j}\left(x\left(k-\tau_{i}\right), x\left(k-\tau_{j}\right)\right)=\sum_{p=0}^{P} \alpha_{p i j} \cdot x\left(k-\tau_{i}\right)\left|x\left(k-\tau_{j}\right)\right|^{p}
$$




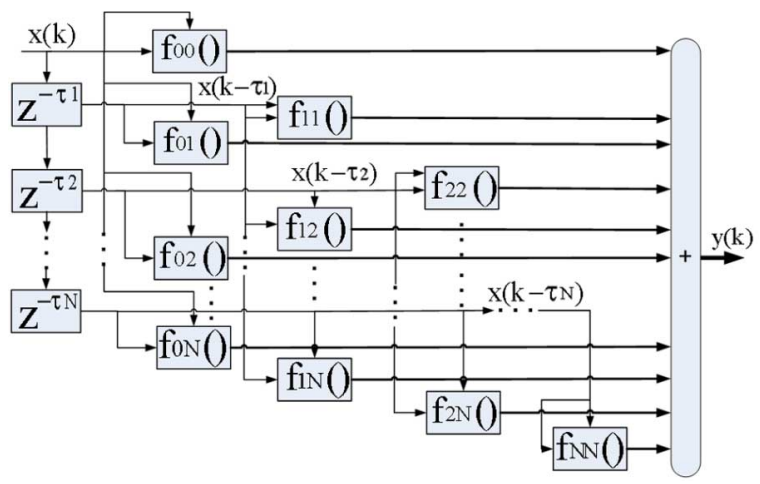

Fig. 1. Augmented nonlinear moving average model structure.

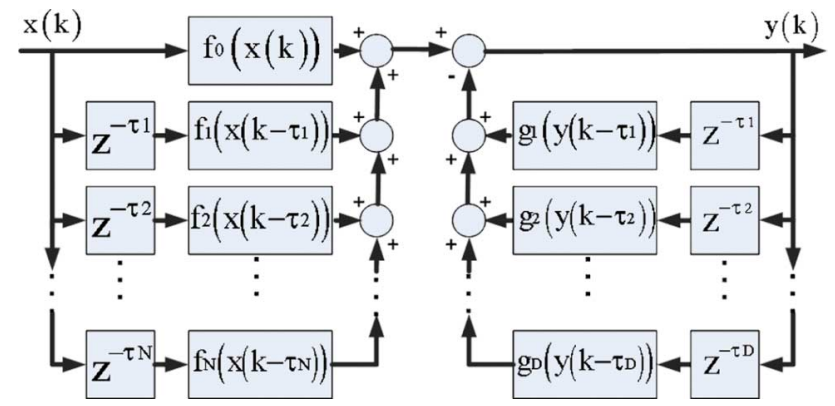

Fig. 2. Nonlinear auto-regressive moving average model structure.

The cost function to be minimized in order to extract the PA model parameters is the normalized square error (NSE). Due to its quadratic nature, this function is also referred as energy function

$$
J(k)=|\bar{e}(k)|^{2}=\left|\left(y(k)-\hat{y}_{\text {Model }}(k)\right) / y(k)\right|^{2} .
$$

\section{B. Nonlinear Auto-Regressive Moving Average Models}

The nonlinear auto-regressive moving average (NARMA) model takes into account both PA's input and output delayed samples $\left(x\left(k-\tau_{i}\right), y\left(k-\tau_{j}\right)\right)$. This kind of structure can reduce the number of coefficients needed to represent short and long term memory effects, but it can also become instable due to the nonlinear $\left(g_{i}\right)$ infinite impulse response (IIR) terms. Therefore, a prior stability test, explained in [1], must be carried out in order to assure its inherent stability.

The general structure of a NARMA model is shown in Fig. 2 and its output signal can be expressed as

$$
\hat{y}_{\text {NARMA }}(k)=\sum_{i=0}^{N} f_{i}\left(x\left(k-\tau_{i}\right)\right)-\sum_{j=1}^{D} g_{j}\left(y\left(k-\tau_{j}\right)\right)
$$

where $f_{i}(\cdot), g_{j}(\cdot)$ are nonlinear functions that can be implemented using polynomial series

$$
\begin{aligned}
f_{i}\left(x\left(k-\tau_{i}\right)\right) & =\sum_{p=0}^{P} \alpha_{\mathrm{pi}} \cdot x\left(k-\tau_{i}\right)\left|x\left(k-\tau_{i}\right)\right|^{p} \\
g_{j}\left(y\left(k-\tau_{j}\right)\right) & =\sum_{p=0}^{P} \beta_{p j} \cdot y\left(k-\tau_{j}\right)\left|y\left(k-\tau_{j}\right)\right|^{p} .
\end{aligned}
$$

As presented before, the cost function to be minimized in order to extract the parameters of the PA models is described in (3).

\section{Heuristic AlgorithMS}

\section{A. Simulated Annealing}

Simulated annealing is a quite straightforward random-search technique based on the Metropolis Monte Carlo (MMC) method and has been proven to be very useful when dealing with nonlinear problems [5]. One of the major advantages of this technique over other methods is its ability to avoid becoming trapped in local minima. The simulated annealing algorithm not only accepts changes that decrease the cost (or energy) function, but also some changes that increase it, accepted with a probability (p)

$$
p=e^{-\delta E / T}
$$

where $\delta E$ is the increase in the energy function and $T$ is a control parameter called temperature.

Focusing in our particular minimization problem it is necessary to define:

1) a representation of possible solutions, e.g., values of the delays considered $(\tau \subset N)$ from 1 to " $M$ " delays;

2) a random generator of $N$ or $N+D$ [depending on the PA model, see (1) and (4)] array of possible delays ( $\left.\tau^{\prime} s\right)$;

3 ) an energy function $(E)$ that evaluates the normalized mean square error (NMSE, mean value of the NSE) achieved with a particular solution;

4) an annealing schedule, that is, the initial temperature $(T)$ and rules for lowering it as the search progresses.

Once these considerations have been taken into account, the simulated annealing algorithm is ready to run and search for the optimal combinations of delays that minimize the cost or energy function. Further details on the simulated annealing principles are perfectly explained in [5].

\section{B. Genetic Algorithms}

Genetic Algorithms search procedures are based on the mechanisms of natural selection and natural genetics. A basic genetic algorithm is composed by three operators: reproduction, crossover, and mutation, extensively explained in [6]. By means of these three operators a cost function is iteratively minimized. After several steps the best combination of delays survives and results in a global minimization of the considered cost function.

The search process to find the best delays that contribute at minimizing the NMSE using genetic algorithms differ from the one used in simulated annealing. By using the simulated annealing algorithm it is possible to search for the best $N$ or $N+D$ delays at once. On the other hand, when applying the genetic algorithms no more than one delay can be evaluated at once. Therefore, we first search for the best delay for a particular PA model, and later, fixing this delay as a good solution, we run the genetic algorithms to find the second best delay that contributes, together with the first delay already found, at minimizing the cost function of the PA models.

\section{RESUlTS}

In order to extract PA behavioral models a WCDMA excitation signal with a PAPR of $10 \mathrm{~dB}$ was used. The signal bandwidth and the channels frequency spacing were $\mathrm{BW}=$ $3.84 \mathrm{MHz}$ and $f_{\text {spacing }}=5 \mathrm{MHz}$. The DUT was measured at the 


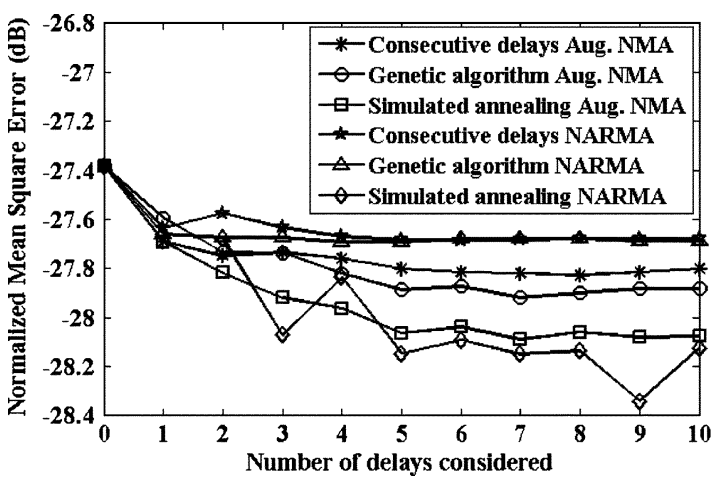

Fig. 3. NMSE (dB) versus number of considered delays.

TABLE I

RESUlts OF POWER AMPLIFIER BeHAVIORAL MODELS

\begin{tabular}{|c|c|c|c|}
\hline $\begin{array}{c}\text { Behavioral } \\
\text { models }\end{array}$ & $\mathbf{N}^{\circ}$ Delays & NMSE (dB) & ACEPR (dB) \\
\hline $\begin{array}{c}\text { Augmented } \\
\text { NMA }\end{array}$ & $\mathbf{5}$ input delays & $\mathbf{- 3 4 . 8 0}$ & $\begin{array}{c}\text { Lower: } \mathbf{- 4 8 . 6 7} \\
\text { Upper: -46.37 }\end{array}$ \\
\hline NARMA & $\begin{array}{c}\mathbf{3}(1 \text { input and } 2 \\
\text { output delays })\end{array}$ & $\mathbf{- 3 3 . 6 2}$ & $\begin{array}{c}\text { Lower: } \mathbf{- 4 8 . 6 4} \\
\text { Upper: } \mathbf{- 4 6 . 2 7}\end{array}$ \\
\hline
\end{tabular}

mildly nonlinear region (IBO $\sim 5 \mathrm{~dB}$ ). The PA used for measurements is a LDMOS class $\mathrm{AB}$ main amplifier: frequency range of $1.93 \ldots 1.99 \mathrm{GHz}$, maximum output power of $+48 \mathrm{dBm}$ and $38 \mathrm{~dB}$ Gain. The PA's output signal was measured at a center frequency of $1.96 \mathrm{GHz}$ using an Agilent Performance Spectrum Analyzer (PSA) and an Agilent 89611 Vector Signal Analyzer (VSA). The measurement results were processed by the use of the Agilent 89604 distortion test suit.

Fig. 3 shows the NMSE achieved in PA behavioral model identification versus the number of delays considered for both NARMA and augmented NMA models when using three types of delay arrays: consecutive delays, delays obtained from a genetic algorithm and from a simulated annealing search, respectively. For this comparison only $1 \%$ of samples of the measured normalized WCDMA modulated data have been used. It was shown that it is possible to obtain better NMSE for both PA models using a few proper chosen delays provided by the simulated annealing algorithm.

Table I shows the NMSE and the adjacent channel error power ratio (ACEPR, described in [3]), for both PA behavioral models. For the extraction and validation processes two different sets of measured data $\left(10^{5}\right.$ samples each set) were used, respectively. These figures of merit (FOMs) have been obtained considering the first significant minimum number of delays in the simulated annealing search shown in Fig. 3. In Fig. 4 we can see an excellent match between the PA measured output spectrum and the output spectra of the augmented NMA and NARMA models respectively. Fig. 5 shows the limiting curves of the AM/AM-conversion of the reference and both modeled output signals. These limiting curves represent the upper and lower boundary covering the AM/AM-conversion plot, and thus it is possible to compare the amount of memory effects introduced by both models.

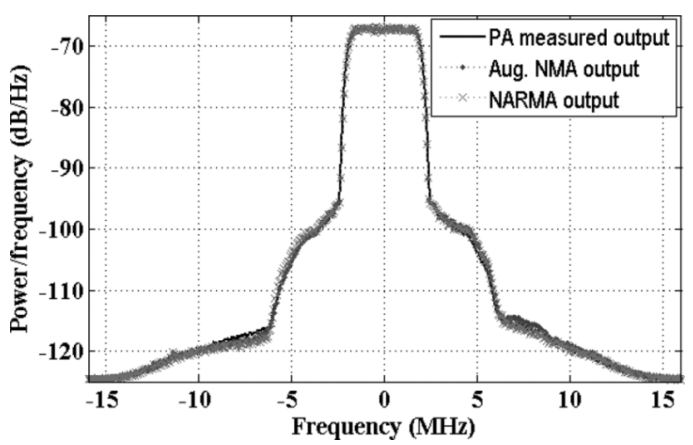

Fig. 4. Measured, augmented NMA and NARMA power spectra.

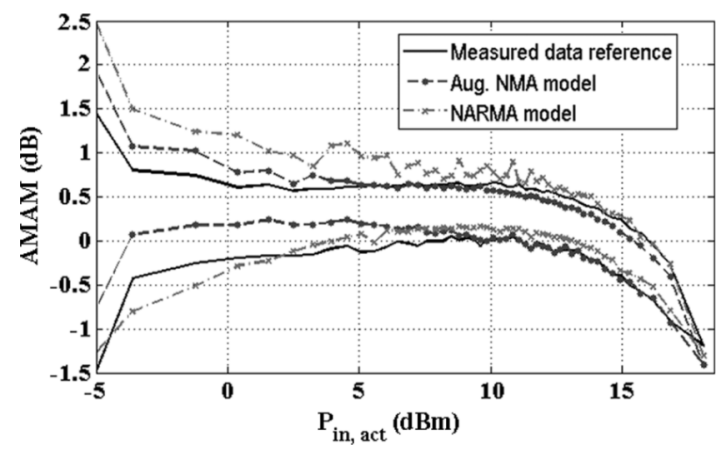

Fig. 5. AM/AM-conversion limiting curves.

\section{CONCLUSION}

Simulated annealing performance has shown better results than genetic algorithms. The reason can be related to our particular configuration of the genetic algorithms search process. The simulated annealing algorithm has shown to be a powerful heuristic search technique that permits to find the minimum and best sparse delays. This contributes to reduce the PA behavioral models complexity, permitting more efficient digital predistortion. Finally, the augmented NMA and the NARMA models provide good FOM results and can be seriously considered for digital predistortion.

\section{REFERENCES}

[1] P. L. Gilabert, G. Montoro, and A. Cesari, "A recursive digital predistorter for linearizing RF power amplifiers with memory effects," in Proc. APMC'06, Dec. 2006, vol. 2, pp. 1043-1047.

[2] J. C. Pedro and S. A. Maas, "A comparative overview of microwave and wireless power-amplifier behavioral modeling approaches," IEEE Trans. Microw. Theory Tech., vol. 53, no. 4, pp. 1150-1163, Apr. 2005.

[3] M. Isaksson, D. Wisell, and D. Ronnow, "A comparative analysis of behavioral models for RF power amplifiers," IEEE Trans. Microw. Theory Tech., vol. 54, no. 1, pp. 348-359, Jan. 2006.

[4] B. O'Brien, J. Dooley, A. Zhu, and T. J. Brazil, "Estimation of memory length for RF power amplifier behavioral models," in Proc. EUMC'06, Sep. 2006, pp. 680-682.

[5] E. Aarts and J. Korst, Simulated Annealing and Boltzmann Machines. A Stochastic Approach to Combinatorial Optimization. New York: Wiley, 1989.

[6] D. E. Goldberg, Genetic Algorithms in Search, Optimization \& Machine Learning. Reading, MA: Addison-Wesley, 1989. 